\title{
Time resolved photoluminescence study of magnetic CdSe/CdMnS/CdS core/multi-shell nanoplatelets
}

Joseph R. Murphy, Savas Delikanli, Tianmu Zhang, Thomas A. Scrace, Peiyao Zhang, et al.

Joseph R. Murphy, Savas Delikanli, Tianmu Zhang, Thomas A. Scrace, Peiyao Zhang, Tenzin Norden, Tim Thomay, Alexander N. Cartwright, Hilmi Volkan Demir, Athos Petrou, "Time resolved photoluminescence study of magnetic CdSe/CdMnS/CdS core/multi-shell nanoplatelets," Proc. SPIE 10114, Quantum Dots and Nanostructures: Growth, Characterization, and Modeling XIV, 101140R (20 February 2017); doi: 10.1117/12.2252266 


\title{
Time resolved Photoluminescence study of magnetic CdSe/CdMnS/CdS core/multi-shell Nanoplatelets
}

\author{
Joseph R. Murphy ${ }^{\mathrm{a}}$, Savas Delikanlib,c, Tianmu Zhang ${ }^{\mathrm{a}}$, Thomas A. Scrace ${ }^{\mathrm{a}}$, Peiyao Zhang ${ }^{\mathrm{a}}$, \\ Tenzin Norden ${ }^{\mathrm{a}}$, Tim Thomay ${ }^{\mathrm{a}}$, Alexander N. Cartwright ${ }^{\mathrm{a}}$, Himli Volkan Demir ${ }^{\mathrm{b}, \mathrm{c}}$, and Athos \\ Petrou $^{\mathrm{a}}$ \\ aState University of New York at Buffalo, Buffalo, NY, USA \\ ${ }^{\mathrm{b} B i l k e n t}$ University, Turkey \\ ${ }^{\mathrm{c}}$ Nanyang Technological University, Singapore
}

\begin{abstract}
Colloidal semiconductor nanoplatelets (NPLs) are quasi 2D-nanostructures that are grown and processed inexpensively using a solution based method and thus have recently attracted considerable attention. We observe two features in the photoluminescence spectrum, suggesting two possible recombination channels. Their intensity ratio varies with temperature and two distinct temperature regions are identified; a low temperature region $(10 \mathrm{~K}<T<90 \mathrm{~K})$ and a high temperature region $(90 \mathrm{~K}<T<200 \mathrm{~K})$. This ratio increases with increasing temperature, suggesting that one recombination channel involves holes that are weakly localized with a localization energy of $0.043 \mathrm{meV}$. A possible origin of these localized states are energy-variations in the $x y$-plane of the nanoplatelet. The presence of positive photoluminescence circular polarization in the magnetically-doped core/multi-shell NPLs indicates a hole-dopant exchange interaction and therefore the incorporated magnetic Manganese ions act as a marker that determines the location of the localized hole states. ${ }^{1}$ Time-resolved measurements show two distinct timescales $\left(\tau_{\text {fast }}\right.$ and $\left.\tau_{\text {slow }}\right)$ that can be modeled using a rate equation model. We identify these timescales as closely related to the corresponding recombination times for the channels. The stronger hole localization of one of these channels leads to a decreased electron-hole wave function overlap and thus a decreased oscillator strength and an increased lifetime. We show that we can model and understand the magnetic interaction of doped 2D-colloidal nanoplatelets which opens a pathway to solution processable spin controllable light sources.
\end{abstract}

\section{INTRODUCTION}

Semiconductor nanocrystals show characteristics that are strongly dependent on both their chemical composition and size. ${ }^{2}$ During the last 40 years, 2D structures were grown as quantum wells by either using molecular beam epitaxy $(\mathrm{MBE})^{3}$ or chemical vapor deposition $(\mathrm{CVD})^{4}$ techniques. However, the 2D structures discussed here are grown using solution-based methods. These colloidal quantum wells, or nanoplatelets (NPLs), are synthesized with a thickness control at the monolayer (ML) level. Whereas the lateral dimensions are large compared to the de Broglie wavelength causing negligible quantum confinement effects in the lateral plane. Single material nanoplatelet core structures using a variety of II-VI semiconductor materials have recently been synthesized by several groups. ${ }^{5-9}$ Very recently, nanoplatelet heterostructures have also been successfully synthesized. ${ }^{10-13}$

The optical properties of zero-dimensional colloidal nanocrystals differ from colloidal nanoplatelets as follows: (a) NPL emission is spectrally narrower ${ }^{14}$ but still tunable, ${ }^{15}$ (b) NPLs show less fluorescence blinking behavior, ${ }^{13}$ and thus (c) NPLs exhibit higher photoluminescence quantum yields. ${ }^{14}$

Further author information: (Send correspondence to T.T.)

T.T.: E-mail: timthoma@buffalo.edu

Quantum Dots and Nanostructures: Growth, Characterization, and Modeling XIV,

edited by Diana L. Huffaker, Holger Eisele, Proc. of SPIE Vol. 10114, 101140R

(c) 2017 SPIE · CCC code: 0277-786X/17/\$18 - doi: 10.1117/12.2252266

Proc. of SPIE Vol. 10114 101140R-1 


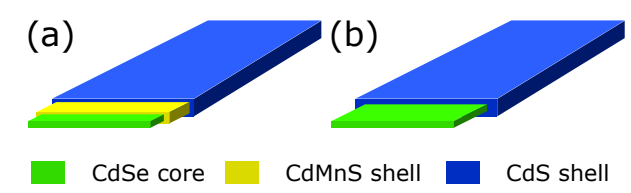

Figure 1. Schematic diagram of a core/multi-shell NPL structure with a CdSe core followed by a single monolayer shell of $\mathrm{CdMnS}$ and terminating with a monolayer of CdS (a) and a CdSe core followed by a shell of two monolayers of CdS (b).

\section{METHODS}

Samples The nanoplatelets used in this study are synthesized such that the thickness, normal to the NPL plane ( $z$-axis), is highly controlled while the lateral size ( $x$ - and $y$-axes) distribution is reasonably uniform. ${ }^{16}$ The dimensions along the $z$-axis in NPLs are comparable to those of QWs grown by conventional epitaxial methods and therefore exhibit similar confinement effects. This confinement in the $z$-direction in NPLs allows for the tailoring of photon emission characteristics by controlling the number of monolayers in each platelet. This can be understood in a similar way that the optical properties of colloidal quantum dots are controlled by adjusting their size. ${ }^{17}$ Nanoplatelets are synthesized colloidally by preparing a NPL solution in a way similar to that used for colloidal quantum dots. Details of the synthesis of these NPLs are given by Delikanli et. al. ${ }^{18}$ Magnetic ions such as manganese are introduced into the layers of our II-VI based NPLs to explore the effects of $s p$ - $d$ exchange interactions between the spins of the carriers and those of the magnetic ions. ${ }^{18}$ The effects of exchange interactions can be tailored through the precise control of the heterostructure composition and therefore the confinement and the lateral dimension. It is possible to precisely control the wave function overlap by controlling the thickness of the component layers and the deposition of one or more magnetic layers through structural design. And thus the overlap of the carrier wave functions with the magnetic regions can be controlled so that the NPLs exhibit specific magneto-optical properties, such as PL circular polarization and Zeeman splitting.

The samples discussed in this proceeding, summarized in table 1, were fabricated according to the procedure described by Delikanli et. al. ${ }^{18}$ by first synthesizing the NPL cores and enveloping this core with successive shell layers using colloidal atomic layer deposition (c-ALD). Core/shell NPL structures were prepared by performing c-ALD deposition on either 5 ML (sample 1, sample 3) or 3 ML (sample 2) seed CdSe cores. During the c-ALD deposition, each side of the CdSe core surface was coated with 1 ML of shell material of either $\mathrm{Cd}_{0.985} \mathrm{Mn}_{0.015} \mathrm{~S}$ or CdS. As a result, for each cycle of shell growth, a thickness of 2 MLs total was deposited with 1 ML each on the top and bottom surface. A schematic of the NPL heterostructures is shown figure 1. Non-magnetic sample 3 was used as a reference sample for the magnetic samples 1 and 2. The latter two (sample 1 and 2) were studied for the investigation of exchange interactions between the spins of $\mathrm{Mn}^{2+}$ ions and those of the carriers.
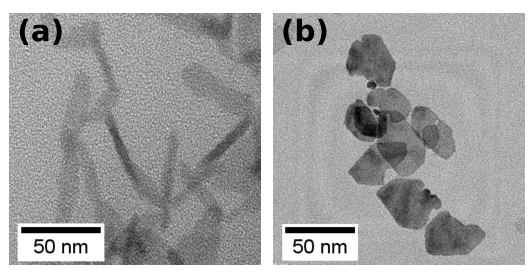

Figure 2. High-angle annular dark-field transmission electron microscopy (HAADF-TEM) images of sample 1 (left), and sample 2 (right).

High-angle annular dark-field transmission electron microscopy (HAADF-TEM) images of magnetic samples 1 (2), are shown figure 2 left (right). These images show that the 5 ML CdSe seed NPLs used as cores of sample 1 exhibit regular rectangular shapes with average lateral dimensions of $55 \pm 6 \mathrm{~nm} \times 10 \pm 2 \mathrm{~nm}$.

The dimensions of the 3 ML CdSe cores of sample 2 are difficult to determine as they tend to fold and thus have more irregular shapes. To determine the manganese concentration of the magnetic shell layers in samples 1 
Table 1. Samples used in this study, the values in parentheses indicate the number of monolayers along the $z$-axis.

\begin{tabular}{|c|c|}
\hline Sample & Composition \\
\hline 1 & CdSe (5) / CdMnS (2) / CdS (2) \\
\hline 2 & CdSe (3) / CdMnS (2) / CdS (2) \\
\hline 3 & CdSe (5) / CdS (2) \\
\hline
\end{tabular}

and 2, energy-dispersive X-ray spectroscopy (EDS) was used which assumes uniform manganese ion distribution with each CdMnS shell.

\subsection{Experimental Methods}

For photoluminescence (PL) and magneto-PL measurements, the NPLs were excited using the linearly polarized laser light at $405 \mathrm{~nm}$ with a maximum power density of $0.2 \mathrm{~W} / \mathrm{cm}^{2}$. The experiments were conducted in Faraday geometry in which the applied magnetic field is parallel to the direction of the emitted light. Using a combination of a quarter-wave plate and linear analyzer placed before the spectrometer entrance slit the emitted PL was analyzed into its $\sigma_{+}$(left circularly polarized) and $\sigma_{-}$(right circularly polarized) components .

\section{RESULTS}

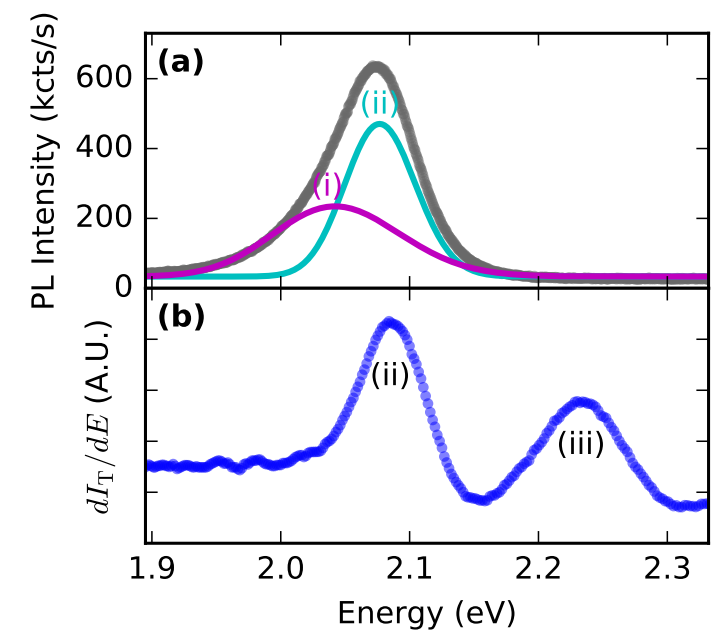

Figure 3. (a) The zero-field PL spectra of the magnetic sample is shown as gray points, result of fitting this data to two Gaussian functions that represent a low-energy (magenta line) and a high-energy (cyan line) component are indicated by (i) and (ii), respectively. (b) The corresponding derivative of the transmission data is shown in blue points with features (ii) also marked, the highest energy transmission feature (iii) is attributed to recombination involving light holes.

In the upper panel of figure 3 we show the zero-field PL spectrum from sample 1 (gray points) at a temperature of $T=10 \mathrm{~K}$. The spectrum shows a pronounced asymmetry on the low-energy side. For this reason, it is fit to the sum of two Gaussian functions centered at $2.036 \mathrm{meV}$ and $2.077 \mathrm{meV}{ }^{1}$ We use a magenta (cyan) line to indicate the low-energy (i) (high-energy (ii)) feature. In figure 3b we plot the spectral derivative of the transmitted intensity, which we have also measured under the same conditions, as a function of photon energy. Two distinct features can be identified: feature (ii) at $2.036 \mathrm{eV}$ coincides with the corresponding PL feature and feature (iii) at $2.233 \mathrm{eV}$. This feature can be identified as the excitonic transition involving the light hole subband. ${ }^{18}$ However, we note that PL feature (i) has no corresponding signature in the transmission spectrum and thus why we attribute feature (i) to a non-excitonic transition. Whereas we attribute feature (ii) to an excitonic transition that involves the lowest heavy hole subband.

In figure 4a (4) we show the two circularly polarized PL components from sample 1 at $B=0 \mathrm{~T}$ and at $B=7$ $\mathrm{T}$ at a temperature of $7 \mathrm{~K}$. Red points indicate the $\sigma_{+}$(LCP) PL component, while the black points show the 


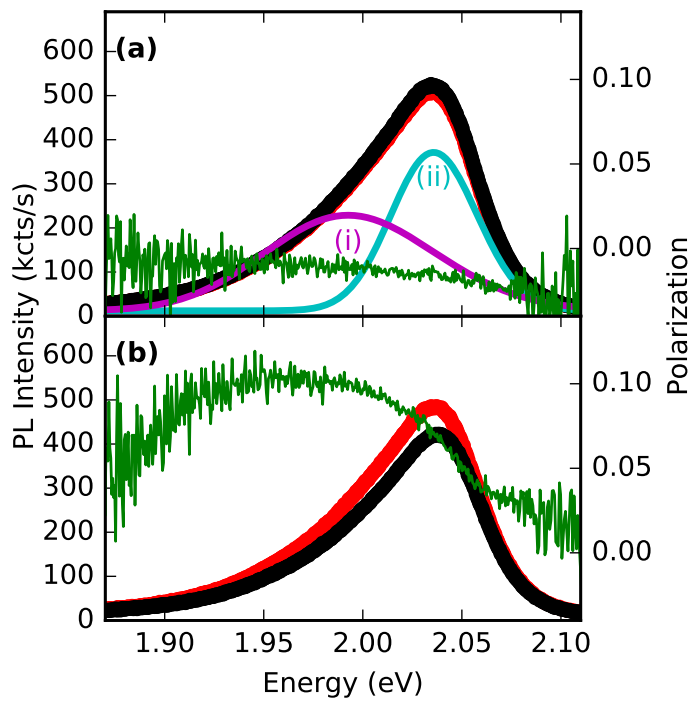

Figure 4. PL spectra from magnetic sample 1 using an excitation wavelength of $405 \mathrm{~nm}$ at a temperature of $7 \mathrm{~K}$. The red (black) points indicate $\sigma_{+}\left(\sigma_{-}\right)$circularly polarized PL components. The green line is the calculated circular polarization. In the upper panel, the applied magnetic field is $0 \mathrm{~T}$ and in the lower panel it is $7 \mathrm{~T}$. The magenta and cyan lines in panel (a) are the result of a fitting that uses two Gaussian functions to the asymmetric PL spectra.

$\sigma_{-}(\mathrm{RCP}) \mathrm{PL}$ component. The circular polarization at a particular photon energy is given by

$$
P=\left(I_{+}-I_{-}\right) /\left(I_{+}+I_{-}\right)
$$

where $I_{+}\left(I_{-}\right)$is the intensity of the $\sigma_{+}\left(\sigma_{-}\right)$PL component. The spectral dependence of the calculated circular polarization is shown in green. The circular polarization $P$ becomes positive as the applied magnetic field is increased, indicating the presence of exchange interaction between the spins of the carriers and those of the $\mathrm{Mn}^{2+}$ ions. ${ }^{19}$ The polarization peak in figure $4 \mathrm{~b}$ shows a maximum that coincides with the intensity maximum of feature (i) which indicates that the corresponding recombination occurs in the vicinity of the manganese ions located in the CdMnS shells.

Comparing figures 4 and 5 the PL line asymmetry is also observed in the non-magnetic sample 3 . In figure 5 (a) and 5(b) we show the two circularly polarized PL components at $B=0 \mathrm{~T}$ and $B=7 \mathrm{~T}$ measured at $7 \mathrm{~K}$. Red points indicate the $\sigma_{+}$(LCP) PL component, while the black points denote the $\sigma_{-}$(RCP) PL component. However, the overall circular polarization of the PL emission from sample 3 is negative and furthermore, unlike sample 1, it shows no distinct spectral features.

In figure 6 the net PL circular polarization, determined by integrating the spectral intensities of the $\sigma_{+}$and $\sigma_{-}$PL components, is plotted as a function of applied magnetic field at a temperature of $7 \mathrm{~K}$. For magnetic sample 1 (blue squares) and sample 2 (yellow triangles), $P$ initially increases with field and saturates before 4 T. For sample 2 however, the polarization saturation value is larger than that of sample 1 due to an increased carrier wave function overlap with the CdMnS shell. ${ }^{18}$ The polarization saturation values of both samples 1 and 2 decrease dramatically with increasing $T$ and vanish at approximately $25 \mathrm{~K}$. We conclude that the CdMnS shells exhibit Brillouin paramagnetism as the circular polarization follows the same dependence on $B$ and $T$ as the magnetization. ${ }^{19}$ As shown in figure 6 the magnetic field dependence of the polarization for the non-magnetic sample 3 (green diamonds) is distinctly different from that of the magnetic samples 1 and 2. Sample 3 shows a negative circular polarization and has a nearly linear dependence on magnetic field. We attribute the negative sign of the circular polarization for sample 3 to the intrinsic conduction and valence band $g$-factors of CdSe. ${ }^{20}$

The origin of the circular polarization in the $\mathrm{Pl}$ of the magnetic samples 1 and 2 is due to the difference in the population distribution of the spin $\pm \frac{1}{2}$ electrons and the spin $\pm \frac{3}{2}$ holes among the conduction and valence band states. This imbalance is due to the individual Zeeman splitting in the conduction and valence band populations. 


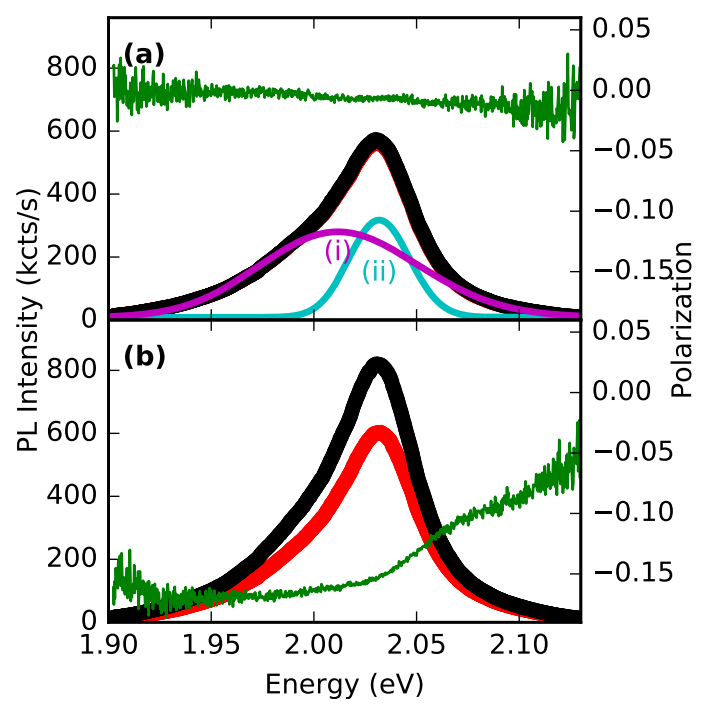

Figure 5. PL spectra from non-magnetic sample 3 using an excitation wavelength of $405 \mathrm{~nm}$ at a temperature of $7 \mathrm{~K}$. The red (black) points indicate $\sigma_{+}\left(\sigma_{-}\right)$circularly polarized PL components. The green line is the calculated circular polarization. In the upper panel, the applied magnetic field is $0 \mathrm{~T}$ and in the lower panel it is $7 \mathrm{~T}$. The magenta and cyan lines in panel (a) are the result of a fitting that uses two Gaussian functions to the asymmetric PL spectra.

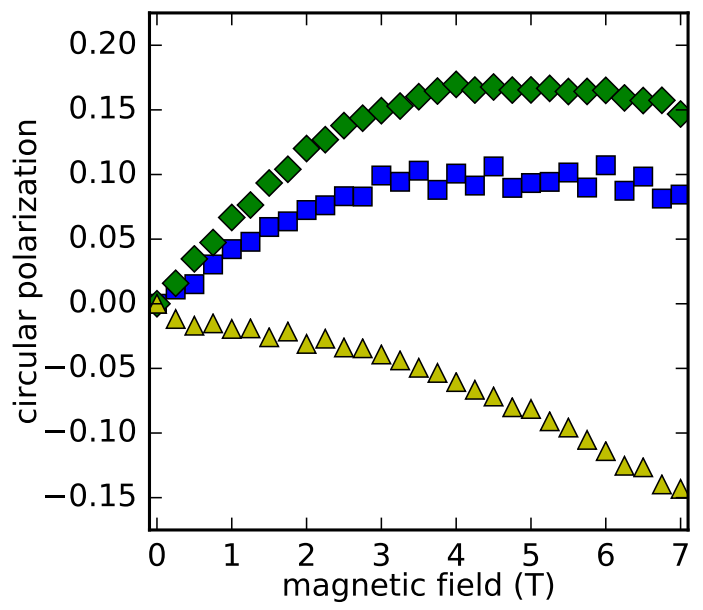

Figure 6. Circular polarization $P$ plotted as a function of magnetic field at a temperature of $T=7 \mathrm{~K}$. Data for sample 1 is indicated by the blue squares, sample 2 is indicated by yellow triangles and sample 3 is indicated by green diamonds.

The net Zeeman splitting, $\Delta E_{\mathrm{Z}}$, is defined as

$$
\Delta E_{\mathrm{Z}}=E_{+}-E_{-}
$$

where $E_{+}$and $E_{-}$are the energy of the $\sigma_{+}$and $\sigma_{-}$PL components. The magnetic field dependence of $\Delta E_{\mathrm{Z}}$ in figure 7 is similar to the dependence of $P$ on $B$ shown in figure 6 . The sign of $\Delta E_{\mathrm{Z}}$ as defined above, is compatible with the sign of the polarization. The $\sigma_{+}$component of magnetic samples 1 and 2 has a lower energy than the $\sigma_{-}$component resulting in $I_{+}>I_{-}$and therefore positive circular polarization $P>0$. The opposite is true for the non-magnetic sample 3 where the $\sigma_{-}$component has a lower energy and thus $I_{-}>I_{+}$resulting in negative circular polarization $P<0$.

The dependence of $P$ and $\Delta E_{\mathrm{Z}}$ on magnetic field and temperature for samples 1 and 2 shows the presence of exchange interactions between the carrier spins and those of the $\mathrm{Mn}^{2+}$ ions in the CdMnS shells. The intensity ratio $I_{i i} / I_{i}$ of the two PL features identified in figure 3 is plotted as a function of temperature in figure $8(\mathrm{a})$. This ratio remains nearly unchanged for temperatures between $10 \mathrm{~K}$ and $90 \mathrm{~K}$. However, this ratio increases 


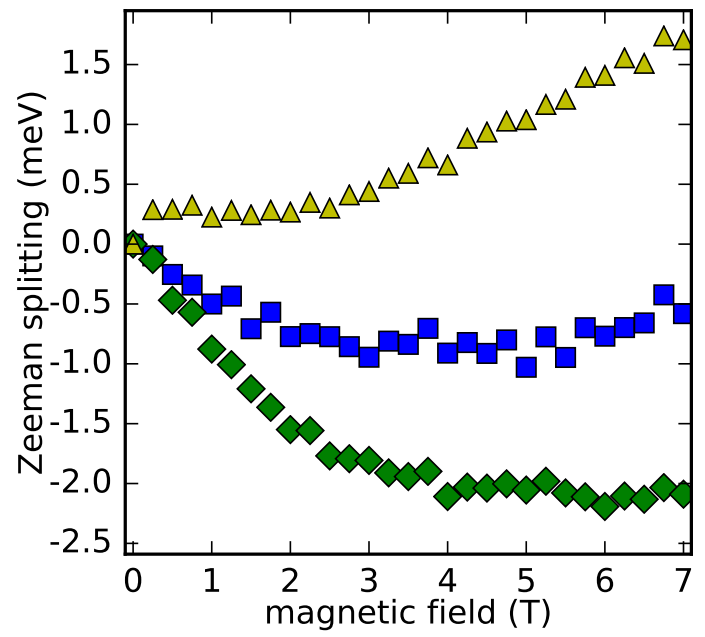

Figure 7. Zeeman splitting of the PL plotted as a function of magnetic field at a temperature of $T=7 \mathrm{~K}$. Datapoints for sample 1 are indicated by the blue squares, sample 2 is indicated by green diamonds; and sample 3 is indicated by yellow triangles.
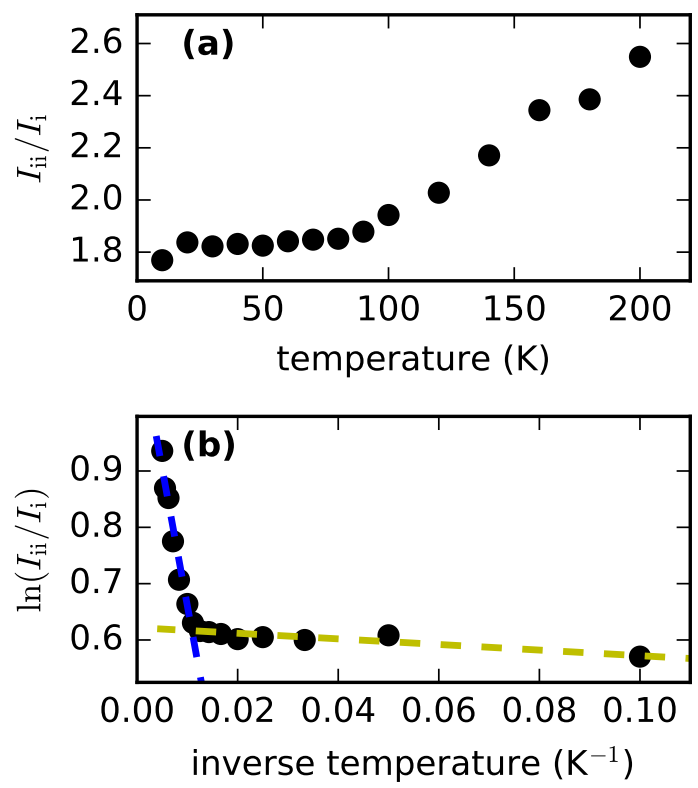

Figure 8. (a) The evolution of the ratio $I_{\mathrm{ii}} / I_{\mathrm{i}}$ as a function of temperature for sample 1 is indicated by the black points. (b) The value of $\ln \left(I_{\mathrm{ii}} / I_{\mathrm{i}}\right)$ is plotted versus $1 / T$; here, $I_{\mathrm{i}}$ and $I_{\mathrm{ii}}$ represent the respective fitted PL peak intensities. Two temperature regions are identified and linear fits to the high (low) temperature data are indicated by the blue (yellow) dashed line.

sharply with increasing temperature for the temperature region between $90 \mathrm{~K}$ and $200 \mathrm{~K}$. In figure $8 \mathrm{~b}$ we plot the $\ln \left(I_{i i} / I_{i}\right)$ as a function of $1 / T$. Using the equation $\ln \left(I_{i i} / I_{i}\right)=-E_{\mathrm{loc}} / k_{\mathrm{B}} T$ we can extract values for $E_{\mathrm{loc}}$, which is a value for the localization energy associated with the carriers involved in the recombination process of transition (i). For the low temperature region $(10 \mathrm{~K} \leq T \leq 90 \mathrm{~K})$, the value of $E_{\text {loc }}$ is very small, indicating the presence of a band of closely spaced energy levels. In contrast, $E_{\text {loc }}$ in the high-temperature region $(90 \mathrm{~K} \leq T \leq 200 \mathrm{~K})$ is equal to $4.2 \mathrm{meV}$.

Based on the results of figure 8, we are able to propose a model of the band structure and the recombination mechanisms associated with PL features (i) and (ii). As mentioned earlier, feature (ii) is excitonic in nature, whereas feature (i) involves recombination of delocalized electrons with hole states localized at the CdSe/CdMnS 


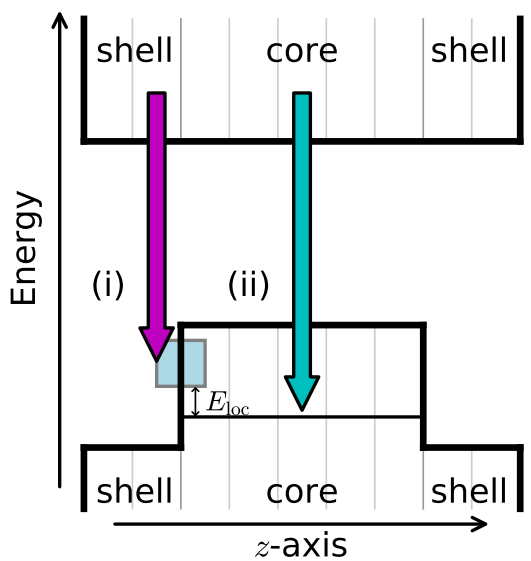

Figure 9. A schematic diagram of the recombination scheme of our core/multi-shell nanoplatelet heterostructures. The vertical axis represents energy and the horizontal axis the position along the vertical $z$-dimension. The spacing between the grid of light grey lines indicates the thickness of single monolayers. The two proposed recombination channels are indicated by the magenta and cyan arrows marked (i) and (ii) corresponding to the PL features identified in figure 3.

core/shell interfaces. This finding is strongly supported by the large positive circular polarization associated with feature (i) in the magnetic samples 1 and 2 in the presence of an externally applied magnetic field. Given the fact that the exchange interaction between the spins of holes and the spins of $\mathrm{Mn}^{2+}$ ions is by a factor of 5 stronger than the corresponding electron- $\mathrm{Mn}^{2+}$ interaction, ${ }^{19}$ we conclude that the localized carriers that are involved in the recombination process of feature (i) must be in the vicinity of the magnetic ions and that those carriers therefore are holes. The localization energy, $E_{\mathrm{loc}}$, of the holes is equal to $4.2 \mathrm{meV}$ as discussed above as and as shown in figure 8(b). A possible source of the localized states band shown in figure 9 as the light blue region is the variation of the localized holes states energy in the $x y$-plane of the nanoplatelets at the core/shell interfaces due to irregularities.

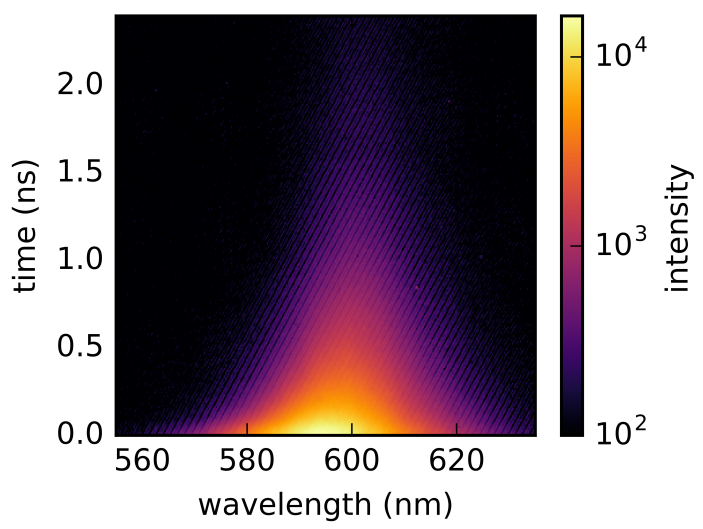

Figure 10. The intensity of the PL is represented by the color of this time resolved PL image, which is mapped in two dimensions. The horizontal axis corresponds to the wavelength in $\mathrm{nm}$ and the vertical axis corresponds to the time delay $\Delta t$ as measured from the arrival of the excitation pulse.

We studied the recombination dynamics in our samples using time resolved PL spectroscopy to determine the origin of these two PL features. In figure 10 we show a 2D time-wavelength map of the PL intensity for the magnetic sample. For each time slice $(\Delta t=10 \mathrm{ps})$, the spectra are fit to a Gaussian function. Examples of these slices are shown in the insets of figures 11. In these insets, the horizontal axis corresponds to energy in $\mathrm{eV}$ and the vertical axis shows a normalized intensity. The spectra shown as insets correspond to times of 0 ns (cyan) 
and $3.5 \mathrm{~ns}$ (magenta). We note that these trPL spectra do not exhibit the same degree of asymmetry as those of the time integrated PL spectra.
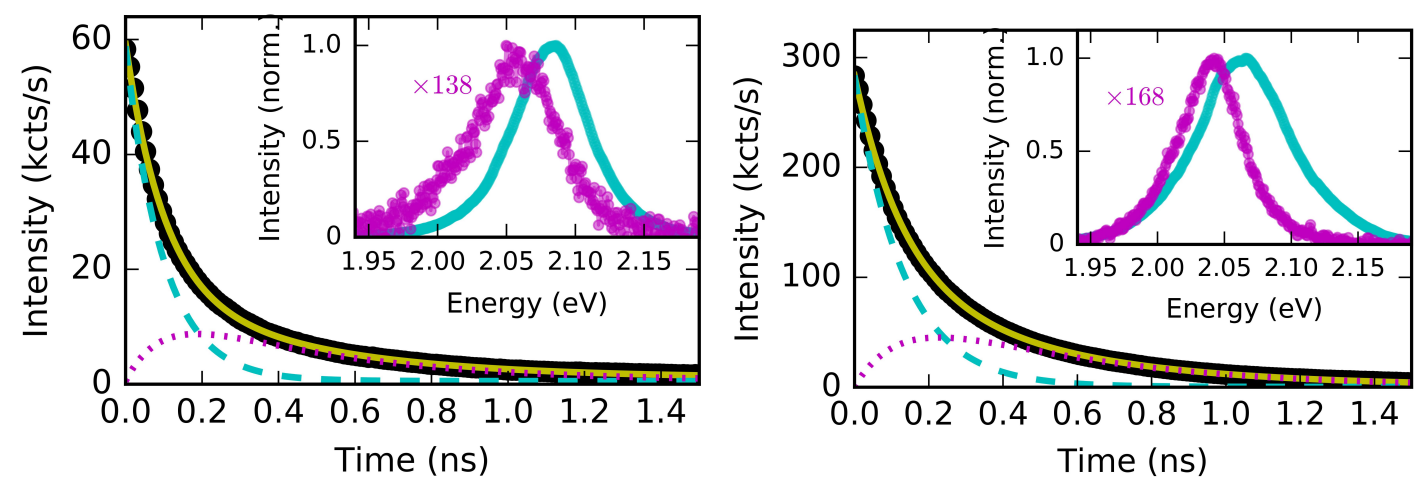

Figure 11. The temporal evolution of the PL intensity from the magnetic sample is shown in the left panel and that of the non-magnetic sample is shown in the right panel using black points. The calculated intensities of features (i) and (ii) are shown using dotted green and dashed blue lines, respectively. The sum of these intensities is indicated by red lines. The data of the insets represent time slices of the trPL spectra at $\Delta t=0 \mathrm{~ns}$ (cyan) and at $\Delta t=3.5$ ns (magenta). These inset spectra have been normalized and dark count-corrected so as to have the same peak intensity, and the magenta numbers indicate the magnification factor for the spectra at $\Delta t=3.5 \mathrm{~ns}$.

the two distinct recombination channels shown in figure 9 correspond to the two PL emission components labeled (i) and (ii) in figure 3. Using a bi-exponential decay function to fit the PL spectra assumes two independent processes with no energy transfer. However to incorporate a coupling of the two processes we constructed a rate equation-based model. To simplify the solutions of these rate equations, we assume the following:

- The time resolution of the streak measurements in this study exceeds the excitation time of the carriers. Therefore, we treat the excitation of electron-hole pairs to be instantaneous. While there is a finite timescale associated with excitation, it is substantially shorter than the resolution.

- The energy transfer occurs only from the high-energy channel (ii) to the low-energy channel (i). The transfer in the opposite direction, i.e., from (i) to (ii), is negligible because the energy difference of those two processes is much greater than $k_{\mathrm{B}} T$, given the low temperature at which this experiment was conducted.

- The result of photo-excitation is that primarily only channel (ii), which is excitonic, is initially populated and channel (i) is empty at $t=0$. The latter becomes populated via energy transfer from channel (ii) as indicated in figure 12 .

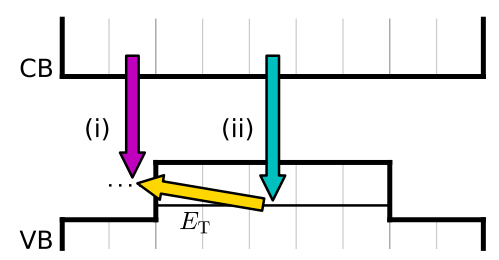

Figure 12. Schematic diagram of the recombination channels shown in figure 4. Here the transfer of excitation from the excitonic channel (ii) to the interface-related channel (i) indicated by the yellow arrow is included.

Based on these assumptions, we construct the following system of coupled differential equations: 
Table 2. Fit parameters of the time-resolved PL intensity using the solutions of the rate equation model.

\begin{tabular}{|c|c|c|}
\hline & Sample 1 & Sample 2 \\
\hline$\beta_{\mathrm{i}}(n s)$ & 0.422 & 0.449 \\
$\beta_{\mathrm{ii}}(n s)$ & 0.098 & 0.131 \\
$C$ & 0.940 & 0.902 \\
\hline
\end{tabular}

$$
\begin{aligned}
\frac{d N_{\mathrm{i}}(t)}{d t} & =-\frac{N_{\mathrm{i}}(t)}{\beta_{\mathrm{i}}}+C \frac{N_{\mathrm{ii}}(t)}{\beta_{\mathrm{i}}} \\
\frac{d N_{\mathrm{ii}}(t)}{d t} & =-\frac{N_{\mathrm{ii}}(t)}{\beta_{\mathrm{ii}}}
\end{aligned}
$$

Here, $N_{\mathrm{i}}(t)$ is the number of carrier pairs at time $t$ associated with channel (i), $N_{\mathrm{ii}}(t)$ is the number of excitons at time $t$ associated with channel (ii), $\beta_{\mathrm{i}}$ is the lifetime associated with channel (i), $\beta_{\mathrm{ii}}$ is the lifetime associated with channel (ii), and $C$ is a parameter that describes the coupling between the two channels.

The analytical solutions to this model are:

$$
\begin{array}{r}
N_{\mathrm{i}}(t)=\frac{1}{\beta_{\mathrm{i}}-\beta_{\mathrm{ii}}}\left(\beta_{\mathrm{i}} N_{\mathrm{i}}(0) e^{-\frac{t}{\beta_{\mathrm{i}}}}-\beta_{\mathrm{ii}} N_{\mathrm{i}}(0) e^{-\frac{t}{\beta_{\mathrm{i}}}}+C \beta_{\mathrm{ii}} N_{\mathrm{ii}}(0) e^{-\frac{t}{\beta_{\mathrm{i}}}}-C \beta_{\mathrm{ii}} N_{\mathrm{ii}}(0) e^{-\frac{t}{\beta_{\mathrm{ii}}}}\right) \\
N_{\mathrm{ii}}(t)=N_{\mathrm{ii}}(0) e^{-\frac{t}{\beta_{\mathrm{ii}}}}
\end{array}
$$

Using the assumption that the state associated with channel (i) is initially unpopulated, the solutions simplify to:

$$
\begin{array}{r}
N_{\mathrm{i}}(t)=\frac{C \beta_{\mathrm{ii}} N_{\mathrm{ii}}(0)}{\beta_{\mathrm{i}}-\beta_{\mathrm{ii}}}\left(e^{-\frac{t}{\beta_{\mathrm{i}}}}-e^{-\frac{t}{\beta_{\mathrm{ii}}}}\right) \\
N_{\mathrm{ii}}(t)=N_{\mathrm{ii}}(0) e^{-\frac{t}{\beta_{\mathrm{ii}}}}
\end{array}
$$

The value of $N_{\mathrm{ii}}(0)$ was set to the PL intensity at $t=0$ and the remaining parameters: $\beta_{\mathrm{i}}, \beta_{\mathrm{ii}}$ and $C$ were used to fit these solutions to the experimentally obtained data. Values for $\beta_{\mathrm{i}}$ and $\beta_{\mathrm{ii}}$ were constrained to be non-negative and values of $C$ were constrained to be within the interval $[0,1]$.

The results of the fit for the magnetic (non-magnetic) sample are summarized in figure 11. In the main panels of figure 11a and 11b, we plot the following: calculated PL intensity of channel (i) using dotted magenta lines; calculated PL intensity of channel (ii) using dashed cyan lines; calculated PL intensity of both channels using yellow lines (which is the sum of the magenta and cyan lines) and the experimental PL intensities integrated over all recorded wavelengths using black points.

The resulting fit parameters are summarized in table 2. Our approach is to fit the solutions to the integrated spectral intensity over all recorded wavelengths as shown in figure 11a and figure 11b. The difference in the values obtained for $\beta_{\mathrm{i}}$ and $\beta_{\mathrm{ii}}$ is compatible with the fact that channel (ii) is excitonic in nature while channel (i) is due to recombination between free electrons with localized holes. We note that the values of the extracted lifetimes in particular that of the slow process, (i), while sensitive to the underlying assumptions, always result in two distinct timescales. Of the lifetimes associated with these processes the lower-energy process is significantly slower than that of the high-energy process. We identify the parameters $\beta_{\mathrm{i}}$ and $\beta_{\mathrm{ii}}$ listed in table 2 as the lifetimes associated with the respective channels. 


\section{CONCLUSIONS}

We have studied the magneto photoluminescence of solution-grown nanoplatelets which consist of CdSe cores, followed by magnetically doped CdMnS shells and terminated with CdS shells. The net PL emission becomes predominantly left circularly polarized $\left(\sigma_{+}\right)$indicating the presence of exchange interaction between the spins of the carriers and those of the $\mathrm{Mn}^{2+}$ ions in the presence of an externally applied magnetic field. The sign of the Zeeman splitting is compatible with the circular polarization of the PL emission, i.e., $E_{+}<E_{-}$, where $E_{+}$ $\left(E_{-}\right)$is the energy associated with the recombination of spin $\mp \frac{1}{2}$ electrons with spin $\pm \frac{3}{2}$ holes. We identified a Brillouin-like dependence on magnetic field and temperature for both the circular polarization and the Zeeman splitting. The time integrated photoluminescence emission is found to be asymmetric and can be decomposed into two distinct Gaussian features. The circular polarization maximum coincides with the intensity peak of the low-energy feature. Using the $\mathrm{Mn}^{2+}$ ions as a marker, we identified this low-energy feature as a recombination of delocalized electrons with holes localized at the CdSe/CdMnS interface. The high-energy feature is excitonic in nature and involves recombination of delocalized electrons with holes confined in the CdSe core. The presence of two distinct lifetimes, $\beta_{\mathrm{i}}$ and $\beta_{\mathrm{ii}}$, determined from the analysis of trPL experimental results further supports this suggested model.

\section{Acknowledgment}

The authors would like to thank EU-FP7 Nanophotonics4Energy NoE, and TUBITAK EEEAG 109E002, 109E004, 110E010, 110E217, and 112E183, and NRF-RF-2009-09, NRF-CRP-6-2010-02, and A*STAR of Singapore for the financial support. H.V.D. acknowledges support from ESF-EURYI and TUBA-GEBIP. Work at the University at Buffalo was supported by NSF DMR 1305770.

\section{REFERENCES}

[1] Murphy, J. R., Delikanli, S., Scrace, T., Zhang, P., Norden, T., Thomay, T., Cartwright, A. N., Demir, H. V., and Petrou, A., "Time-resolved photoluminescence study of cdse/cdmns/cds core/multi-shell nanoplatelets," Applied Physics Letters 108(24), 242406 (2016).

[2] Alivisatos, A. P., "Perspectives on the physical chemistry of semiconductor nanocrystals," The Journal of Physical Chemistry 100(31), 13226-13239 (1996).

[3] Dingle, R., "Confined carrier quantum states in ultrathin semiconductor heterostructures," in [Festkörperprobleme 15], Queisser, H., ed., Advances in Solid State Physics 15, 21-48, Springer Berlin Heidelberg (1975).

[4] Dupuis, R. D., Dapkus, P. D., Jr., N. H., Rezek, E., and Chin, R., "Roomtemperature laser operation of quantumwell ga(1x)alxasgaas laser diodes grown by metalorganic chemical vapor deposition," Applied Physics Letters 32(5), 295-297 (1978).

[5] Ithurria, S. and Dubertret, B., "Quasi 2D colloidal cdse platelets with thicknesses controlled at the atomic level," Journal of the American Chemical Society 130(49), 16504-16505 (2008). PMID: 19012381.

[6] Ithurria, S., Tessier, M. D., Mahler, B., Lobo, R. P. S. M., Dubertret, B., and Efros, A. L., "Colloidal nanoplatelets with two-dimensional electronic structure," Nature Materials 10, 936-941 (2011).

[7] Joo, J., Son, J. S., Kwon, S. G., Yu, J. H., and Hyeon, T., "Low-temperature solution-phase synthesis of quantum well structured cdse nanoribbons," Journal of the American Chemical Society 128(17), 5632-5633 (2006). PMID: 16637619.

[8] Li, Z., Qin, H., Guzun, D., Benamara, M., Salamo, G., and Peng, X., "Uniform thickness and colloidal-stable cds quantum disks with tunable thickness: Synthesis and properties," Nano Research 5(5), 337-351 (2012).

[9] Schliehe, C., Juarez, B. H., Pelletier, M., Jander, S., Greshnykh, D., Nagel, M., Meyer, A., Foerster, S., Kornowski, A., Klinke, C., and Weller, H., "Ultrathin pbs sheets by two-dimensional oriented attachment," Science 329(5991), 550-553 (2010).

[10] Ithurria, S. and Talapin, D. V., "Colloidal atomic layer deposition (c-ald) using self-limiting reactions at nanocrystal surface coupled to phase transfer between polar and nonpolar media," Journal of the American Chemical Society 134(45), 18585-18590 (2012). PMID: 23061923. 
[11] Kelestemur, Y., Olutas, M., Delikanli, S., Guzelturk, B., Akgul, M. Z., and Demir, H. V., "Type-ii colloidal quantum wells: Cdse/cdte core/crown heteronanoplatelets," The Journal of Physical Chemistry C 119(4), 2177-2185 (2015).

[12] Mahler, B., Nadal, B., Bouet, C., Patriarche, G., and Dubertret, B., "Core/shell colloidal semiconductor nanoplatelets," Journal of the American Chemical Society 134(45), 18591-18598 (2012). PMID: 23057684.

[13] Tessier, M. D., Spinicelli, P., Dupont, D., Patriarche, G., Ithurria, S., and Dubertret, B., "Efficient exciton concentrators built from colloidal core/crown cdse/cds semiconductor nanoplatelets," Nano Letters 14(1), 207-213 (2014). PMID: 24328730.

[14] Tessier, M. D., Mahler, B., Nadal, B., Heuclin, H., Pedetti, S., and Dubertret, B., "Spectroscopy of colloidal semiconductor core/shell nanoplatelets with high quantum yield," Nano Letters 13(7), 3321-3328 (2013). PMID: 23731211.

[15] Delikanli, S., Guzelturk, B., Hernandez-Martinez, P. L., Erdem, T., Kelestemur, Y., Olutas, M., Akgul, M. Z., and Demir, H. V., "Continuously tunable emission in inverted type-i cds/cdse core/crown semiconductor nanoplatelets," Advanced Functional Materials 25(27), 4282-4289 (2015).

[16] Guzelturk, B., Olutas, M., Delikanli, S., Kelestemur, Y., Erdem, O., and Demir, H. V., "Nonradiative energy transfer in colloidal cdse nanoplatelet films," Nanoscale 7, 2545-2551 (2015).

[17] Murray, C. B., Norris, D. J., and Bawendi, M. G., "Synthesis and characterization of nearly monodisperse cde (e = sulfur, selenium, tellurium) semiconductor nanocrystallites," Journal of the American Chemical Society 115(19), 8706-8715 (1993).

[18] Delikanli, S., Akgul, M. Z., Murphy, J. R., Barman, B., Tsai, Y., Scrace, T., Zhang, P., Bozok, B., Hernández-Martínez, P. L., Christodoulides, J., Cartwright, A. N., Petrou, A., and Demir, H. V., "Mn2+doped cdse/cds core/multishell colloidal quantum wells enabling tunable carrier-dopant exchange interactions," ACS Nano 9(ja), 12473 (2015). (Web) November 14, 2015.

[19] Furdyna, J. K., "Diluted magnetic semiconductors," Journal of Applied Physics 64(4), R29-R64 (1988).

[20] Long, G., Barman, B., Delikanli, S., Tsung Tsai, Y., Zhang, P., Petrou, A., and Zeng, H., "Carrier-dopant exchange interactions in mn-doped pbs colloidal quantum dots," Applied Physics Letters 101(6), 062410 (2012). 